\title{
Herbal Origin Natural Products are Boon for Human Race
}

\author{
Manish $\mathrm{K}^{1,2 *}$ and Nitin $\mathrm{B}^{2}$ \\ 1PhD Research Scholar, IKG Punjab Technical University, India \\ ${ }^{2}$ Department of Pharmacology, ASBASJSM College of Pharmacy, India
}

*Corresponding author: Manish Kumar, Assistant Professor, Swift School of Pharmacy, Ghaggar Sarai, Rajpura, Patiala (Punjab) 140401, India, mkpharmacology@gmail.com manishkumar@swiftcollegeedu.in

\begin{abstract}
mpharmacology@gmail.com manishkumar@swiftcollegeedu.in
\end{abstract}
\author{
(n)
}

\section{Editorial}

The practice of natural products and phytochemicals in various ailments has apparently long history marked by first clinical use of Colchicum autumnale (autumn crocus) in treatment of gout by Alexander of Tralles in 6th century $\mathrm{AD}$ [1]. Before that, different medical records such as Ebers Papyrus (Egyptian drugs compendium), Sushruta Samhita (Indian herbal drugs record), Chinese Materia Medica, Shennong Herbal and the Tang Herbal compiled several herbal remedies still used in numerous modern pharmacotherapies. The five volumes of De Materia Medica authored by Pedanius Dioscorides (Greek physician) were translated into several languages worldwide owing to the large record of herbal remedies grouped first time in therapeutic categories that subsequently enhanced the basis of pharmacological understanding of diseases [2]. Sushruta Samhita and Charak Samhita form the basis of 5000 years old ancient Indian system of medicine practice popularly known as 'Ayurveda'. Theophrastus (original name was Tyrtamus later changed by Aristotle), is a well acknowledged biologist and Peripatetics, also known as 'father of botany' by scientific community, significantly contributed towards therapeutic activities of plants. Galen was a Greek chemist globally known for his work on plant extracts. Ibn Sina or Avicenna (Persian philosopher) wrote several books such as 'The Book of Healing' and 'The Canon of Medicine (Canon Medicinae)' based on therapeutic utility of plant extracts which later on formed the foundation of Yunani system of medicine [3]. Seydler (German chemist) coined the term 'Pharmacognosy' and authored the book 'Analecta Pharmacognistica' in 1815.

Ancient civilizations efficiently utilized therapeutic efficacy of several herbs in promotion of physical vigour (e.g. Ashwagandha, Shilajit, Shatavari) as well as mental health (e.g. H. perforatum, V. officinalis, Brahmi). Historically the natural products have proven track record in combating morbidity and mortality rates. The use of crude drugs such as poppy seeds (morphine), cinchona bark (quinine) and Azadirachta indica leaves in folklore are testimonial to the withstanding of human race against several epidemics. However, with time the alternative drug therapy witnessed need for more refined products. Subsequently, advancement in molecular techniques, isolation of novel compounds and methods to elucidate their structural properties led to a significant growth in identification of some novel herbal therapeutic molecules such as vincristine (Catharanthus roseus), paclitaxel (Taxus brevifolia) and salicin (Willow tree bark). These isolated molecules served as template or lead compounds for synthesis of potent and therapeutically more specific new chemical entities (NCEs) in drug discovery (e.g. acetylsalicylic acid) [4]. Contemporarily almost every part of Azadirachta indica (popularly known as neem in India) was used in chronic inflammatory and infectious diseases. At present several compounds isolated from neem such as nimbine, nimbidine, azadirachtin have shown potent and specific antibacterial, antimalarial, antioxidant and antiinflammatory properties [5]. This leads to classification of natural products in diverse chemical (e.g. alkaloids, glycosides, tannins, flavonoids) and therapeutic categories which not only enhances pharmacological knowledge but also makes easier for practitioners. Above all, the ultimate goal of all biological research is welfare of human health which needs a direction in present scenario. A comprehensive data of drug dose and associated adverse effects is pivotal in any therapy including herbal medicine practice that can be resolved satisfactorily only through purification of novel compounds. However, several crude herbal drugs such as ginseng or extracts still enjoy wide 
popularity which indicates significance of consumption of certain herbs/part as whole. Preclinical and clinical studies demonstrated immunomodulatory activities of ginseng which prompted global emergence of many herbal immune booster formulations.

The phytochemical diversity provides a natural-pool of therapeutically active molecules rigorously exploited by advanced methods of drug screening (e.g. high-throughput screening) to provide impetus to our quest of NCEs. Quite a few phytochemicals have successfully undergone preclinical studies [6] and are under clinical evaluation. Some are established therapeutic drugs widely prescribed by physicians viz. galantamine, huperzine. Certain crude drugs and extracts also highlight the prescription list exemplified by liquorice, Gingko biloba, St. John's Wort, turmeric tincture. However, many herbs of medicinal importance are near extinction or declared as endangered as a plethora of human activities (e.g. deforestation, global warming) have disturbed their natural habitat. The purified lead compounds can be used in laboratory synthesis of several of these depleting novel molecules or NCEs.

A survey by WHO suggested that $80 \%$ people are using herbal medicinal products globally. The extraction, purification and in vivo bioassay of isolated phytochemicals have enhanced the therapeutic usability and faith in traditional medicinal system. The science of identification, isolation and purification of herbal natural compounds is multi-disciplinary which requires sound knowledge of organic, biological chemistry and analytical techniques. For obtaining high yield appropriate selection of procedure (e.g. extraction, fractionation and chromatography) is quintessential. Some factors considered in selection of proper technique are: (i) amount of desired compound in the crude drug; (ii) the physicochemical characteristic of the compound; (iii) presence of other compounds of similar characteristics poses difficulty in the isolation and purification of desired substance. Contemporarily employed extraction procedures (e.g. solvent, ultrasound, microwave-assisted extraction and supercritical fluid extraction), liquid-solid chromatography (e.g. PPC, column chromatography) and partition chromatographic techniques (e.g. HPLC, VLC, GC) have proven useful, however, the culmination of several challenges lead to multiple-steps purification procedure. The chemical characterization of natural product is accomplished using modern spectroscopy techniques such as NMR (H1 or C13), mass spectroscopy (fourier transform MS, LCMS, GCMS), infrared spectroscopy (FTIR) and UV visible spectroscopy. Subsequently, after the toxicity studies, the biological activities of pure compound is affirmed by in-vitro studies and pre-clinical studies. A successful completion of clinical stages accomplishes the drug discovery process.

Ground breaking discoveries of some natural products in 1945 (penicillin), 1952 (streptomycin) and 2015 (avermectins and artemisinin) were awarded noble prizes in field of physiology/medicine which subsequently carved their own niche in therapeutics [7]. In modern world the definition of natural products (also known as complimentary, traditional or alternative medicines) is broad but in a simple way can be referred to a remedy obtained from any biological source such as animals, plants or micro-organisms (e.g. antibiotics). However, a majority of natural products include crude herbal drugs and isolated phytochemicals (secondary metabolites), vitamins, minerals and several plant origin dietary supplements which may be categorised into pharmaceuticals, cosmeceuticals or nutraceuticals. Majority of alternative systems of medicinal practice such as ayurveda, homeopathy, aromatherapy, naturopathy and traditional Chinese medicinal system utilize plant derivatives in some form or another. At present the drug regulatory agencies (US FDA, Health Canada and European Medicines Agency) are facing challenges regarding quality, supply, dose and toxicity of crude herbal drugs (extracts) which potentiate need of purified therapeutically active compounds. It is very pertinent to state that natural products have devastating effect on human race since antiquity and holds a promising future.

\section{References}

1. Nuki G, SimKin PA (2006) A concise history of gout and hyperuricemia and their treatment. Arthritis Res Ther 8(1): S1.

2. Dias DA, Urban S, Roessner U (2012) A historical overview of natural products in drug discovery. Metabolites 2(2): 303-336.

3. Naini FB (2012) Avicenna and the Canon Medicinae. JRSM 105(4): 142.

4. Krause J, Tobin G (2013) Discovery, development, and regulation of natural products in Using Old solutions to new problems - natural drug discovery in the 21st century, Kulka M (Ed.) InTech.

5. Alzohairy MA (2016) Therapeutics role of azadirachta indica (neem) and their active constituents in diseases prevention and treatment. Evid Based Complement Alternat Med 2016: 7382506. 
6. Shen B (2015) A new golden age of natural products drug discovery. Cell 163(6): 1297-1300.
7. Kumar M, Bansal N (2018) Caffeic acid phenethyl ester rescued streptozotocin-induced memory loss through PI3-kinase dependent pathway. Biomed Pharmacother 101: 162-173. 